\title{
The influence of high-density lipoprotein cholesterol on maximal lipid core burden indexing thin cap fibrous atheroma lesions as assessed by near infrared spectroscopy
}

\author{
Magdalena M. Dobrolińska ${ }^{1}$, Paweł Gąsior ${ }^{1}$, Wojciech Wańha ${ }^{1}$, \\ Przemysław Pietraszewski ${ }^{2}$, Elżbieta Pociask ${ }^{3}$, Grzegorz Smolka ${ }^{1}$, \\ Wojciech Wojakowski ${ }^{1}$, Tomasz Roleder ${ }^{4}$
}

${ }^{1}$ Division of Cardiology and Structural Heart Diseases, Medical University of Silesia in Katowice, Poland ${ }^{2}$ Department of Sports Theory, Jerzy Kukuczka Academy of Physical Education in Katowice, Poland

${ }^{3}$ Department of Biocybernetics and Biomedical Engineering,

AGH University of Science and Technology, Krakow, Poland

${ }^{4}$ Regional Specialist Hospital, Research and Development Center, Wroclaw, Poland

\begin{abstract}
Background: Previous studies suggest that higher plasma concentrations of several lipid molecules are associated with higher lipid core burden index (LCBI) near infrared spectroscopy (NIRS) imaging. The aim of this study was to investigate whether an association between plasma lipids depends on plaque morphology (thin cap fibrous atheroma [TCFA] vs. non-TFCA) as measured by near-infrared spectroscopy-intravascular ultrasound (NIRS-IVUS).

Methods: Sixty-four patients retrospectively enrolled were diagnosed with stable coronary artery disease or acute coronary syndrome who underwent NIRS-IVUS imaging. Before percutaneous coronary intervention, blood samples were collected for measurement of serum levels of total cholesterol (TC), lowdensity lipoprotein cholesterol (HDL-C), high-density lipoprotein cholesterol (HDL-C) and triglycerides (TG). Patients were divided into two groups based on maxLCBI ${ }_{4 m m}$ and IVUS imaging. Those with maxLCBI ${ }_{4 m m} \geq 323$ were included into TCFA group $(n=35)$ while others were assigned to the non-TCFA $\operatorname{group}(n=29)$.

Results: Thin cap fibrous atheroma lesions were significantly longer than the non-TCFA lesions (25.66 \pm 9.56 vs. $17.03 \pm 9.22, p=0.001)$. TCFA characterizes greater plaque burden $(78.4[70.9,82.2] v s$. 72.70 [64.77, 76,05]; $p=0.021)$ and plaque volume $(176.1[110.75,247.5]$ vs. $68.1[55.58,143.35]$; $p=0.000$ ) as compared to non-TCFA. In TCFA suspected lesions, there was no correlation between max$L_{C B I_{4 m m}}$ and LDL levels $(r=0.105, p=0.549)$ nor TC levels $(r=-0.035, p=0.844)$ but a negative correlation was found between $H D L-C$ and $\operatorname{maxLCBI}_{4 m m}(r=-0.453, p=0.007)$.

Conclusions: The present study showed that there was no correlation between plasma LDL-C, TC and $T G$ level and the amount of lipids in coronary plaque assessed by NIRS in both TCFA and non-TCFA groups. Only HDL-C correlated with maxLCBI ${ }_{4 m m}$ in TCFA lesions. (Cardiol J 2021; 28, 6: 887-895) Key words: lipid plasma levels, NIRS-IVUS
\end{abstract}

Address for correspondence: Tomasz Roleder, MD, PhD, Provintional Specialist Hospital, Research and Development Center, ul. H.M. Kamieńskiego 73a 51-124 Wrocław, Poland, tel: +48 884096034, e-mail: tomaszroleder@gmail.com 


\section{Introduction}

Stable coronary artery disease (SCAD) is a common cause of death. According to the European Heart Network Report from 2017 Ischemic Heart Disease (IHD) is responsible for 862,000 deaths a year (19\% of all deaths) among men and 877,000 deaths (20\%) among women in Europe each year [1]. Untreated SCAD may be followed by acute coronary syndrome (ACS). In most cases (approximately 75\%) ACS is caused by a rupture or erosion of the vulnerable atherosclerotic plaque which leads to an occlusion of the vessel [2-4]. Vulnerable plaques are considered to be thin cap fibrous atheroma (TCFA) [5, 6]. TCFAs were defined by Burke et al. [7] as a necrotic and lipidrich core plaque covered with fibrous cap $<65 \mu \mathrm{m}$ measured by optical coherence tomography (OCT). The lipid-rich core containing plaques can also be detected by near-infrared spectroscopy (NIRS) $[8,9]$, a catheter-based intravascular imaging device based on diffuse reflectance spectroscopy. Its adjustment to intravascular ultrasound (IVUS) enables the periprocedural analysis of the chemical composition of plaque and distinguishes lipid-rich lesions. The amount of lipids is measured as a lipid core burden index $\left({ }_{\max } \mathrm{LCBI}_{4 \mathrm{~mm}}\right)$. Roleder et al. [10] and Inaba et al. [11] identified TCFA as lesions with ${ }_{\max } \mathrm{LCBI}_{4 \mathrm{~mm}} \geq 323$.

High cholesterol levels are strongly associated with SCAD [12-14]. It is known that there is a positive relation between low-density lipoprotein cholesterol (LDL-C) and SCAD $[15,16]$. In contrast, there is an inverse relationship between high-density lipoprotein cholesterol (HDL-C) and the risk of SCAD $[17,18]$. It was established that both LDL-C [19] and HDL-C [20] are associated with plaque volume. However, the influence of lipid plasma levels on the chemical composition of the plaque measured by NIRS remains unknown.

The aim of this study was to investigate whether an association between plasma lipids depends on plaque morphology (TCFA vs. non-TFCA) as measured by NIRS-IVUS.

\section{Methods}

\section{Study population}

All patients diagnosed with SCAD or ACS between 2012 and 2015 who underwent NIRS-IVUS imaging were screened for inclusion in this study. The SCAD and ACS management met the criteria of the European Society of Cardiology [21]. Patients with stent restenosis as a target lesion, re- nal failure (creatinine $>1.5 \mathrm{mg} / \mathrm{dL}$ ), hemodynamic compromise and contrast allergy were excluded from the study. The percutaneous coronary intervention (PCI) was performed under angiography guidance, and NIRS-IVUS data were not used for this purpose. Analyzed segments were targeted in the imaged artery by NIRS-IVUS. None of the patients developed any complications related to NIRS-IVUS imaging.

Before or after the PCI blood samples were collected from every patient on day 1 , after fasting conditions for the measurement of serum levels of total cholesterol (TC), LDL-C, HDL-C and triglyceride (TG). All of them were measured enzymatically using standard methods. Clinical demographics and medical history were obtained from hospital records. The study group consisted of 64 lesions analyzed in 64 patients. Patients were divided into two groups based on ${ }_{\text {max }} \mathrm{LCBI}_{4 \mathrm{~mm}}$ results. Those with ${ }_{\max } \mathrm{LCBI}_{4 \mathrm{~mm}} \geq 323$ were included in the TCFA group $(\mathrm{n}=35)$ while others were assigned to the nonTCFA group $(\mathrm{n}=29)$. The study conformed to the Declaration of Helsinki. Due to retrospective design of the study, further application was not needed.

\section{NIRS-IVUS analysis}

The NIRS-IVUS analysis was performed in culprit lesions using standard protocol before stent implantation. All NIRS-IVUS examinations were performed after heparin anticoagulation (activated clotting time $>250 \mathrm{~s}$ ) and following intracoronary nitroglycerine $(100-200 \mu \mathrm{m})$ administration. The 2.4 Fr. TVC Insight Catheter (InfraReDx, TVC Imaging System ${ }^{\mathrm{TM}}$, Burlington, MA, USA) was positioned at least $10 \mathrm{~mm}$ distal to the imaging target lesion. Subsequently, the automated pullback was started with at $0.5 \mathrm{~mm} / \mathrm{s}$ (240 rotations/min) until the TVC catheter entered the guiding catheter. Measurements were made every $1 \mathrm{~mm}$ in region of interest (ROI), and the target lesion was required to be at least $4 \mathrm{~mm}$ long. Quantitative gray-scale IVUS measurements were performed every $1 \mathrm{~mm}$ in the scanned coronary segment before stent implantation. Cross-sectional images were quantified for lumen diameters and area, external elastic lamina (EEM) diameters and area, total plaque area, plaque burden, and lumen and EEM eccentricity by one observer. Plaque burden was calculated as total plaque area divided by EEM CSA $\times 100$ (\%). The remodeling index (RI) was calculated by dividing EEM area at the minimal lumen area (MLA) by the reference EEM area. Lesions with RI $\leq 0.95$ were defined as negatively remodeled, while 
Table 1. Patient characteristics.

\begin{tabular}{|c|c|c|c|}
\hline & TCFA $(n=35)$ & Non-TCFA $(n=29)$ & $\mathbf{P}$ \\
\hline \multicolumn{4}{|l|}{ Clinical demographics } \\
\hline Age [years] & $63.2 \pm 11.37$ & $66.64 \pm 9.8$ & 0.176 \\
\hline Women & $9(25.7 \%)$ & $9(31 \%)$ & 0.637 \\
\hline BMI $\left[\mathrm{kg} / \mathrm{m}^{2}\right]$ & $27.2 \pm 6.07$ & $26.4 \pm 4.95$ & 0.625 \\
\hline Prior MI & $9(25.7 \%)$ & $10(34.5 \%)$ & 0.445 \\
\hline Prior $\mathrm{PCl}$ & $13(37.1 \%)$ & $13(37.1 \%)$ & 0.533 \\
\hline Prior CABG & $3(8.5 \%)$ & $2(6.9 \%)$ & 0.845 \\
\hline Dyslipidemia & $35(100 \%)$ & $29(100 \%)$ & \\
\hline Hypertension & $30(85.7 \%)$ & $27(93.1 \%)$ & 0.209 \\
\hline Diabetes mellitus & $15(42.8 \%)$ & $9(31 \%)$ & 0.331 \\
\hline Statin use & $30(85.7 \%)$ & $25(86.2 \%)$ & 0.719 \\
\hline Hemoglobin & $12.97 \pm 1.64$ & $12.7 \pm 2.5$ & 0.635 \\
\hline Creatinine & $1.06 \pm 0.26$ & $1.01 \pm 0.25$ & 0.514 \\
\hline GFR & $68.62 \pm 18.77$ & $70.7 \pm 16.04$ & 0.767 \\
\hline \multicolumn{4}{|l|}{ Procedural findings } \\
\hline \multicolumn{4}{|c|}{ Indication for coronary angiography } \\
\hline ACS & $8(22.85 \%)$ & $8(27.58 \%)$ & 0.664 \\
\hline Region of interest [mm] & $25.66 \pm 9.56$ & $17.03 \pm 9.22$ & 0.001 \\
\hline \multicolumn{4}{|l|}{ Imaged coronary artery } \\
\hline Left main artery & $1(5.7 \%)$ & 0 & 0.05 \\
\hline Left descending artery & $20(57.1 \%)$ & $11(37.9 \%)$ & 0.05 \\
\hline Circumflex artery & $9(25.7 \%)$ & $5(17.2 \%)$ & 0.05 \\
\hline Right coronary artery & $5(14.3 \%)$ & $13(44.8 \%)$ & 0.05 \\
\hline
\end{tabular}

Variables are displayed as mean \pm standard deviation when a normal distribution is present, or as median ( $1^{\text {st }}-3^{\text {rd }}$ quartile) when there was not a normal distribution present. For each variable, the percentage of patients involved ( $\%$ ) is given. ACS - acute coronary syndrome; $\mathrm{BMI}$ - body mass index; CABG - coronary artery bypass grafting; GFR - glomerular filtration rate; MI — myocardial infarction; $\mathrm{PCl}$ - percutaneous coronary intervention; TCFA - thin cap fibrous atheroma

those with $\mathrm{RI} \geq 1.05$ were defined as positively remodeled. RI between these values was taken as a non-remodeled vessel.

The chemical composition of the plaque in ROI was acquired using NIRS. The lipid-rich plaques are displayed on chemogram on which the $\mathrm{X}$-axis shows the pullback position (1 pixel every $0.1 \mathrm{~mm}$ ) and Y-axis displays the circumferential position ( 1 pixel every $1 \mathrm{~mm}$ ). Based on chemogram the LCBI in $4 \mathrm{~mm}$ was measured. There was a fraction of pixels indicating lipids (yellow pixels) within the ROI. The LCBI with ${ }_{\text {max }} \mathrm{LCBI}_{4 \mathrm{~mm}}$ was measured automatically by NIRS software. TCFA suspected lesions were defined as ${ }_{\text {max }} \mathrm{LCBI}_{4 \mathrm{~mm}} \geq 323$.

NIRS-IVUS data were analyzed off-line using CAAS intravascular software (Pie Medical Imaging BV, Maastricht, The Netherlands).

\section{Statistical analysis}

Normality of variables was assessed by the Kolmogorov-Smirnov test. For normally distributed data values were presented as the mean with a standard deviation ( \pm SD). Non-normally distributed continuous variables were displayed as a median with interquartile intervals (IQR, $\left.1^{\text {st }}, 3^{\mathrm{d}}\right)$. Categorical data were shown as the number or percentage $(\%)$. For the normally distributed group comparison, the one-way ANOVA was used. Non-normally distributed data were compared using the Mann-Whitney test. The correlation was measured using the Pearson correlation coefficient for normally distributed values and the Spearman rank-order correlation for non-normally distributed variables. The categorical data were compared using the Fischer exact test or $\chi^{2}$ test. A value of 
Table 2. Summary of near-infrared spectroscopy-intravascular ultrasound (NIRS-IVUS) results.

\begin{tabular}{lccc}
\hline & TCFA $(\mathbf{n}=35)$ & Non-TCFA $(\mathbf{n}=29)$ & P \\
\hline NIRS analysis & $551.0(423.0,697.0)$ & $137(27.0,232.0)$ & 0.000 \\
max LCBI ${ }_{4 m m}$ & & & 0.001 \\
IVUS analysis & $25.66 \pm 9.56$ & $17.03 \pm 9.22$ & 0.05 \\
Stenosis length & $114.800(86.100,137.90)$ & $75.10(55.75,128.60)$ & 0.003 \\
Lumen volume & $301.6(212.9,393.7)$ & $153.2(105.85,272.1)$ & 0.056 \\
EEM volume & $11.2(9.5,12.6)$ & $10.0(7.35,11.6)$ & 0.000 \\
EEM area at MLA & $176.1(110.75,247.5)$ & $68.1(55.58,143.35)$ & 0.021 \\
Plaque volume & $78.4(70.9,82.2)$ & $72.70(64.77,76,05)$ & 0.69 \\
Plaque burden & $2.57(1.9,3.4)$ & $2.60(2.05,3.22)$ & 0.334 \\
MLA & $1.6(1.5,1.7)$ & $1.60(1.50,1.80)$ & 0.047 \\
MLD & $1.7(1.6,2.0)$ & $1.80(1.65,2.00)$ & 0.69 \\
MLD based on reference & $1.02(0.8,1.27)$ & $1.00(0.84,1.44)$ & \\
Remodeling index & & & \\
\hline
\end{tabular}

Variables are displayed as mean \pm SD when a normal distribution is present, or as median $\left(1^{\text {st }}-3^{\text {rd }}\right.$ quartile) when there was not a normal distribution present. For each variable, the percentage of patients involved ( $\mathrm{n} \%$ ) is given. EEM - external elastic lamina; MLA - minimal lumen area; MLD - minimal lumen diameter

$\mathrm{p}<0.05$ was considered statistically significant. SPSS version 25.0 (SPSS, Inc., Chicago, Illinois) was used for statistical analysis.

\section{Results}

\section{Patients characteristics}

The baseline characteristics are summarized in Table 1. Between 2012 and 201564 patients were enrolled in the study. All patients included underwent PCI followed by stent deployment. Patients from the TCFA group were not-significantly younger than patients from non-TCFA group $(63.2 \pm 11.37$ vs. $66.64 \pm 9.8 ; \mathrm{p}=0.176)$. All patients in both groups had dyslipidemia. There were no significant differences in the percentage of patients who had hypertension $(85.7 \%$ vs. $93.1 \%$, $\mathrm{p}=0.209)$ or diabetes mellitus $(42.8 \%$ vs. $31 \%$, $\mathrm{p}=0.331$ ) in TCFA or non-TCFA groups. At the time of the procedure, the percentage of patients treated with statins was similar in both groups ( $85.7 \%$ vs. $86.2 \%$; $\mathrm{p}=0.719)$. Nine $(25.7 \%)$ of TCFA patients and $10(34.5 \%)$ of non-TCFA patients presented with a history of myocardial infarction $(\mathrm{p}=0.445)$.

\section{IVUS lesion analysis}

Intravascular ultrasound results are reported in Table 2 . The NIRS-IVUS analysis was performed during coronary angiography. TCFA lesions were significantly longer than non-TCFA lesions $(25.66 \pm 9.56$ vs. $17.03 \pm 9.22$; $\mathrm{p}=0.001)$. TCFA characterizes greater plaque burden $(78.4[70.9,82.2]$ vs. $72.70[64.77,76,05]$; $\mathrm{p}=0.021)$ and plaque volume (176.1 [110.75, $247.5]$ vs. 68.1 [55.58, 143.35$] ; \mathrm{p}=0.000)$ as compared to non-TCFA. The values of lumen volume (114.800 [86.100, 137.90] vs. 75.10 [55.75, 128.60]; $\mathrm{p}=0.05)$ and EEM volume $(301.6[212.9,393.7]$ vs. $153.2[105.85,272.1] ; p=0.003)$ were also higher in TCFA group. According to MLA and minimal lumen diameter there were no significant differences comparing both groups (respectively, $\mathrm{p}=0.69, \mathrm{p}=0.334)$. No difference was found in RI for both groups as well $(1.02[0.8,1.27]$ vs. 1.00 $[0.84,1.44] ; \mathrm{p}=0.69)$.

\section{Association between NIRS analysis and lipid plasma levels}

Comparing TCFA to non-TCFA group, the differences in the levels of LDL-C (80.00 [69.00, $111.00]$, vs. 72.00 [58.50, 97.50]; $p=0.43)$, HDL-C (39.50 [35.75, 46.00] vs. 42.00 [33.50, 53.00]; $\mathrm{p}=0.47), \mathrm{TC}(146.00[122.00,181.00]$ vs. 140.00 $[120.50,167.50 ; \mathrm{p}=0.80)$ and $\mathrm{TG}(119.00[74.00$, $160.00]$ vs. 102.00 [83.50, 120.50]; $\mathrm{p}=0.34$ ) were not statistically significant. Also, the number of patients with LDL $>70$ was not significantly higher in TCFA group as compared to non-TCFA group (24 vs. $15 ; p=0.223$ ) (Fig. 1 ). The summary of lipid plasma levels is listed in Table 3.

In non-TCFA group there was no significant correlation between ${ }_{\max } \mathrm{LCBI}_{4 \mathrm{~mm}}$ and LDL levels $(\mathrm{r}=-0.55, \mathrm{p}=0.783)$, TC levels $(\mathrm{r}=-0.133$, $\mathrm{p}=0.498)$ nor HDL-C levels $(\mathrm{r}=-0.062, \mathrm{p}=0.754)$. In TCFA lesions there was also no correlation 
Table 3. Summary of lipid plasma levels.

\begin{tabular}{lccc}
\hline Blood lipid levels & TCFA $(\mathbf{n}=35)$ & Non-TCFA $(\mathbf{n}=29)$ & P \\
\hline LDL-C & $80.00(69.00,111.00)$ & $72.00(58.50,97.50)$ & 0.43 \\
HDL-C & $39.50(35.75,46.00)$ & $42.00(33.50,53.00)$ & 0.47 \\
TC & $146.00(122.00,181.00)$ & $140.00(120.50,167.50)$ & 0.80 \\
TG & $119.00(74.00,160.00)$ & $102.00(83.50,120.50)$ & 0.34 \\
\hline
\end{tabular}

Variables are displayed as mean \pm standard deviation when a normal distribution is present, or as median ( $1^{\text {st }}-3^{\text {rd }}$ quartile) when there was not a normal distribution present. For each variable, the percentage of patients involved ( $\mathrm{n} \%$ ) is given. HDL-C - high density lipoprotein cholesterol; LDL-C — low density lipoprotein cholesterol; TC — total cholesterol; TCFA — thin cap fibrous atheroma; TG - triglycerides

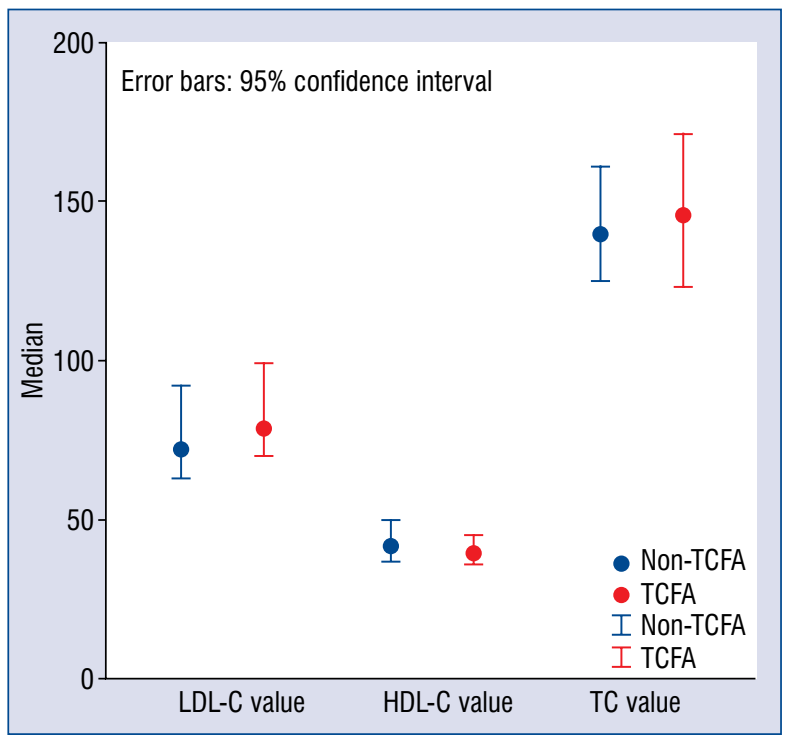

Figure 1. The relation of lipid plasma levels between thin cap fibrous atheroma (TCFA) and non-TCFA group. Variables are displayed as median with $1^{\text {st }}-3^{\text {rd }}$ quartile; HDL-C - high density lipoprotein cholesterol; LDL-C — low density lipoprotein cholesterol; TC - total cholesterol.

between ${ }_{\text {max }} \mathrm{LCBI}_{4 \mathrm{~mm}}$ and LDL levels $(\mathrm{r}=0.105$, $\mathrm{p}=0.549)$ nor TC levels $(\mathrm{r}=-0.035, \mathrm{p}=0.844)$ but a negative correlation was found between HDL-C and ${ }_{\text {max }} \mathrm{LCBI}_{4 \mathrm{~mm}}(\mathrm{r}=-0.453, \mathrm{p}=0.007)$ (Fig. 2).

\section{Discussion}

The major findings of the present study are as follows: 1 ) there was no correlation between LDL-C, TC and TG plasma levels and the number of lipids in coronary plaque assessed by NIRS in both groups, 2) HDL-C correlated with ${ }_{\max } \mathrm{LCBI}_{4 \mathrm{~mm}}$ in TCFA lesions, 3) there was no difference in LDL-C, HDL-C, TC and TG plasma levels in patients with TCFA compared to those with non-TCFA lesions,
4) based on IVUS analysis TCFA characterized greater values of plaque volume, plaque burden and stenosis length.

A large number of patients suffering from SCAD has aroused an interest in the pathology of atherosclerotic plaque. The Dynamic Registry revealed that $6 \%$ of lesions being $<50 \%$ in severity during initial angiography progressed mainly as ACS [22]. It showed that there is a need for a new diagnostic approach to finding clinically silent plaques. Pathological studies have found TCFA lesions to be the main precursor of plaque rupture in patients suffering from ACS $[6,23]$. It was distinguished by a fibrous cap thickness which was $<65 \mu \mathrm{m}$, a small MLA, and a larger plaque burden [6]. This finding and the fact that an angiographic evaluation of the stenosis was not sufficient led to the development of intravascular imaging modalities which facilitated in vivo TCFA identification. Especially OCT imaging, due to its high resolution emerged as a favorable intravascular modality in confirming a vulnerable plaque presence. OCT measurements determined a borderline fibrous cap thickness of $<65 \mu \mathrm{m}[6,7,24]$. Also, studies evaluating multiple imaging modalities enabled TCFA identification using IVUS, NIRS-IVUS or VH-NIRS. IVUS, in contrast to OCT, IVUS enables deep penetration into the plaque. Roleder et al. [24] emphasized its superior role in the estimation of plaque burden, vessel remodeling, and plaque vulnerability identification. Based on its measurements TCFA was defined as a lesion with a plaque burden greater than $70 \%$, and a MLA of $4 \mathrm{~mm}^{2}$ or less [25]. Those lesions were also associated with higher major adverse cardiac event (MACE) rate [26] and positive remodeling [27]. Based on our results, TCFA characterizes greater plaque burden and plaque volume.

The adjustment of NIRS to IVUS enables the periprocedural analysis of chemical composition of the plaque and distinguishes lipid-rich lesions. 


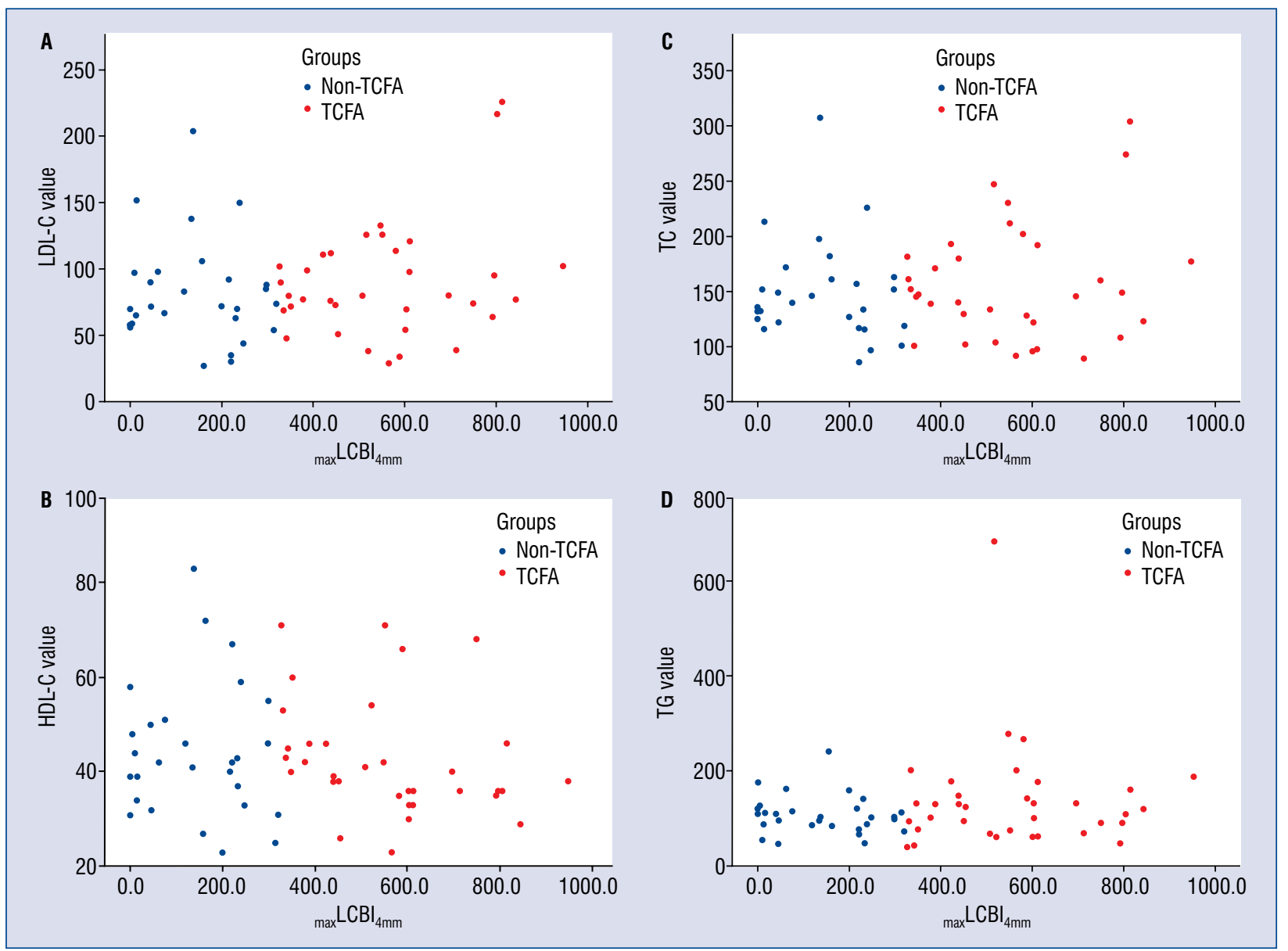

Figure 2. A-D. The correlations between low density lipoprotein cholesterol (LDL-C) (A), high density lipoprotein cholesterol (HDL-C) (B), total cholesterol (TC) (C), triglycerides (TG) (D) and ${ }_{\max } \mathrm{LCBI}_{4 \mathrm{~mm}}$. A. Correlation between LDL-C in thin cap fibrous atheroma (TCFA) and non-TCFA group was respectively $0.105(p=0.549)$ and $-0.55(p=0.783)$; B. Correlation between HDL-C in TCFA and non-TCFA group was respectively $-0.453(p=0.007)$ and $-0.062(p=0.754)$;

C. Correlation between TC in TCFA and non-TCFA group was respectively $-0.035(p=0.844)$ and $-0.133(p=0.498)$;

D. Correlation between TG in TCFA and non-TCFA group was respectively $0.059(p=0.735)$ and $-0.21(p=0.283)$.

It was also believed that identification of lipid-rich plaques based on chemical analysis might point out lesions responsive to intensive lipid-lowering therapy. It not only plays a qualitative role but also enables the quantitative measurement of lipid content as a ${ }_{\max } \mathrm{LCBI}_{4 \mathrm{~mm}}[9,28]$. According to NIRS results, TCFA was described by Inaba et al. [11] as a lesion with a threshold of ${ }_{\max } \mathrm{LCBI}_{4 \mathrm{~mm}}$ $\geq 323$. A statistically significant association between ${ }_{\max } \mathrm{LCBI}_{4 \mathrm{~mm}}$ and an increased incidence of MACE was also observed [29, 30].

It is commonly known that higher LDL-C and TC plasma levels increase the risk of vulnerable plaque [18]. Some clinical trials showed that LDL-C lowering therapy decreases the risk of cardiovascular events. The influence of LDL-C reduction of lipid content measured by NIRS was found in the
Yellow trial which showed a decrease in ${ }_{\max } \mathrm{LCBI}_{4 \mathrm{~mm}}$ after rosuvastatin intensive therapy [31]. Even though LDL-C is considered to be one of the main factors of SCAD, many patients presenting with SCAD have LDL-C within normal levels, which was shown in a study carried out by Sachdeva et al. [32] revealing that only $50.5 \%$ of the patients admitted with SCAD presented LDL-C levels greater than $100 \mathrm{mg} / \mathrm{dL}$ [32]. This may explain the lack of correlation between LDL-C and ${ }_{\text {max }} \mathrm{LCBI}_{4 \mathrm{~mm}}$ for both groups in the present study. According to LDL-C plasma levels in TCFA and non-TCFA group, Nasu et al. [14] showed that in the TCFA group LDL-C levels were significantly higher than in the non-TCFA group while a study conducted by Nagasawa et al. [33] revealed no difference between those groups and this is what was also demonstrated in the current study. 
High density lipoprotein cholesterol is inversely related to SCAD. Its main role is to remove the excessive cholesterol by transporting its particles from non-hepatic cells to the liver and other steroidogenic organs [34]. It is believed that it plays a pivotal role in the inhibition of atherosclerosis by reducing the migration of inflammatory cells into the artery wall and inhibiting LDL-C oxidation [35]. The Framingham Heart Study highlighted HDL-C as a risk factor for SCAD and considered it to be of greater importance as compared to LDL-C [17]. A study conducted by Honda et al. [36] revealed that independent of statin use HDL-C inversely correlated with a change in lipid plaque composition assessed by NIRS. The mentioned study and other studies did not show an association between $\mathrm{LDL}$ and ${ }_{\max } \mathrm{LCBI}_{4 \mathrm{~mm}}$, which indicates the role of HDL-C in plaque composition and also agrees with the findings of the present study [37, 38]. It suggests that HDL-C may decrease the vulnerability of the plaque, but this finding requires further investigation.

As far as TG are concerned, it was shown that they were among factors inducing the plaque progression [39], but are not independently associated with CAD. Further studies focused on the relationship between lipid plasma levels and plaque morphology showed no relation between TG and ${ }_{\max } \mathrm{LCBI}_{4 \mathrm{~mm}}$ which was also found in the current study.

It needs to be highlighted that only periprocedural lipid plasma levels were measured with no insight into long-term analysis. Danek et al. [40] studied the progression of atherosclerosis in saphenous vein grafts and revealed that in contrast to the LDL-C and TG measured annually, the periprocedural measurements showed no association with ${ }_{\text {max }} \mathrm{LCBI}_{4 \mathrm{~mm}}$. It was also found that annual measurements are more representative for the assessment of plaque morphology. It may explain the lack of correlation between lipid plasma levels and ${ }_{\max } \mathrm{LCBI}_{4 \mathrm{~mm}}$ in the present study.

According to patients who had suffered from myocardial infarction in the past, there was no significant difference between the TCFA and nonTCFA group. What was found was that all these patients had a significantly lower ${ }_{\max } \mathrm{LCBI}_{4 \mathrm{~mm}}$ value which had probably occurred due to lipid-lowering therapy.

\section{Limitations of the study}

This study involves a small number of patients which leads to an increased risk of selection bias. The main limitation of this study is the fact that the length of lipid-lowering therapy remains unknown and was not taken into account. Additionally, only the stented lesion was analyzed without insight into other plaques in the coronary tree which may have led to an inaccurate assessment of the association between lipid plasma levels and plaque morphology. A larger and prospective study is needed to verify the results of this study.

\section{Conclusions}

The major finding of the present study showed that there was no correlation between plasma LDL-C, TC, and TG level and the number of lipids in coronary plaque assessed by NIRS in both TCFA and non-TCFA group. Only HDL-C correlated with ${ }_{\max } \mathrm{LCBI}_{4 \mathrm{~mm}}$ in TCFA lesions. There was also no difference in LDL-C, HDL-C, TC, and TG in patients with TCFA compared to those with non-TCFA lesions. Based on IVUS analysis TCFA characterized greater values of plaque volume, plaque burden and stenosis length.

\section{Conflict of interest: None declared}

\section{References}

1. Wilkins E, Wilson L, Wickramasinghe K, et al. European Cardiovascular Disease Statistics 2017 edition. Eur Hear Network, Brussels. 2017, doi: 978-2-9537898-1-2.

2. Farb A, Tang AL, Burke AP, et al. Sudden coronary death. Frequency of active coronary lesions, inactive coronary lesions, and myocardial infarction. Circulation. 1995; 92(7): 1701-1709, doi: 10.1161/01.cir.92.7.1701, indexed in Pubmed: 7671351.

3. Davies MJ, Richardson PD, Woolf N, et al. Risk of thrombosis in human atherosclerotic plaques: role of extracellular lipid, macrophage, and smooth muscle cell content. Br Heart J. 1993; 69(5): 377-381, doi: 10.1136/hrt.69.5.377, indexed in Pubmed: 8518056.

4. Kolodgie FD, Burke AP, Farb A, et al. The thin-cap fibroatheroma: a type of vulnerable plaque: the major precursor lesion to acute coronary syndromes. Curr Opin Cardiol. 2001; 16(5): 285-292, doi: 10.1097/00001573-200109000-00006, indexed in Pubmed: 11584167.

5. Virmani R, Kolodgie F, Burke A, et al. Lessons from sudden coronary death. Arter Thromb Biol. 2000; 20(5): 1262-1275, doi: 10.1161/01.atv.20.5.1262.

6. Virmani R, Burke A, Willerson J, et al. The pathology of vulnerable plaque. Vulnerable Atherosclerotic Plaque. 2007: 19-36, doi: 10.1002/9780470987575.ch2.

7. Burke AP, Farb A, Malcom GT, et al. Coronary risk factors and plaque morphology in men with coronary disease who died suddenly. N Engl J Med. 1997; 336(18): 1276-1282, doi: 10.1056/ NEJM199705013361802, indexed in Pubmed: 9113930.

8. Madder RD, Goldstein JA, Madden SP, et al. Detection by nearinfrared spectroscopy of large lipid core plaques at culprit sites in 
patients with acute ST-segment elevation myocardial infarction. JACC Cardiovasc Interv. 2013; 6(8): 838-846, doi: 10.1016/j. jcin.2013.04.012, indexed in Pubmed: 23871513.

9. Waxman S, Dixon SR, L'Allier P, et al. In vivo validation of a catheter-based near-infrared spectroscopy system for detection of lipid core coronary plaques: initial results of the SPECTACL study. JACC Cardiovasc Imaging. 2009; 2(7): 858-868, doi: 10.1016/j.jcmg.2009.05.001, indexed in Pubmed: 19608137.

10. Roleder T, Kovacic JC, Ali Z, et al. Combined NIRS and IVUS imaging detects vulnerable plaque using a single catheter system: a head-to-head comparison with OCT. EuroIntervention. 2014; 10(3): 303-311, doi: 10.4244/EIJV10I3A53, indexed in Pubmed: 24769522 .

11. Inaba S, Mintz GS, Burke AP, et al. Intravascular ultrasound and near-infrared spectroscopic characterization of thin-cap fibroatheroma. Am J Cardiol. 2017; 119(3): 372-378, doi: 10.1016/j. amjcard.2016.10.031, indexed in Pubmed: 27876264.

12. Silbernagel G, Fauler G, Renner W, et al. The relationships of cholesterol metabolism and plasma plant sterols with the severity of coronary artery disease. J Lipid Res. 2009; 50(2): 334-341, doi: 10.1194/jlr.P800013-JLR200, indexed in Pubmed: 18769018.

13. Strandberg T, Tilvis R, Pitkala K, et al. Cholesterol and Glucose Metabolism and Recurrent Cardiovascular Events Among the Elderly. J Am Coll Cardiol. 2006; 48(4): 708-714, doi: 10.1016/j. jacc.2006.04.081.

14. Nasu K, Terashima M, Habara M, et al. Impact of cholesterol metabolism on coronary plaque vulnerability of target vessels: a combined analysis of virtual histology intravascular ultrasound and optical coherence tomography. JACC Cardiovasc Interv. 2013; 6(7): 746-755, doi: 10.1016/j.jcin.2013.02.018, indexed in Pubmed: 23769651.

15. MacMahon S, Duffy S, Rodgers A, et al. Blood cholesterol and vascular mortality by age, sex, and blood pressure: a metaanalysis of individual data from 61 prospective studies with 55000 vascular deaths. Lancet. 2007; 370(9602): 1829-1839, doi: 10.1016/s0140-6736(07)61778-4.

16. Mihaylova B, Emberson J, Blackwell L, et al. The effects of lowering LDL cholesterol with statin therapy in people at low risk of vascular disease: meta-analysis of individual data from 27 randomised trials. Lancet. 2012; 380(9841): 581-590, doi: 10.1016/S0140-6736(12)60367-5, indexed in Pubmed: 22607822.

17. Gordon T, Castelli WP, Hjortland MC, et al. High density lipoprotein as a protective factor against coronary heart disease. The Framingham Study. Am J Med. 1977; 62(5): 707-714, doi: 10.1016/0002-9343(77)90874-9, indexed in Pubmed: 193398.

18. Sharrett AR, Ballantyne CM, Coady SA, et al. Coronary heart disease prediction from lipoprotein cholesterol levels, triglycerides, lipoprotein(a), apolipoproteins A-I and B, and HDL density subfractions: The Atherosclerosis Risk in Communities (ARIC) Study. Circulation. 2001; 104(10): 1108-1113, doi: 10.1161/ hc3501.095214, indexed in Pubmed: 11535564.

19. Nissen SE, Tuzcu EM, Schoenhagen P, et al. Effect of intensive compared with moderate lipid-lowering therapy on progression of coronary atherosclerosis: a randomized controlled trial. JAMA. 2004; 291(9): 1071-1080, doi: 10.1001/jama.291.9.1071, indexed in Pubmed: 14996776.

20. Nicholls S, Tuzcu E, Sipahi I, et al. Statins, high-density lipoprotein cholesterol, and regression of coronary atherosclerosis. JAMA. 2007; 297(5): 499, doi: 10.1001/jama.297.5.499.

21. Thygesen K, Alpert JS, Jaffe AS, et al. Writing Group on the Joint ESC/ACCF/AHA/WHF Task Force for the Universal Definition of Myocardial Infarction, ESC Committee for Practice Guidelines (CPG). Third universal definition of myocardial infarction. Eur Heart J. 2012; 33(20): 2551-2567, doi: 10.1093/eurheartj/ehs184, indexed in Pubmed: 22922414.

22. Glaser R, Selzer F, Faxon DP, et al. Clinical progression of incidental, asymptomatic lesions discovered during culprit vessel coronary intervention. Circulation. 2005; 111(2): 143-149, doi: 10.1161/01. CIR.0000150335.01285.12, indexed in Pubmed: 15623544.

23. Virmani R, Kolodgie FD, Burke AP, et al. Lessons from sudden coronary death: a comprehensive morphological classification scheme for atherosclerotic lesions. Arterioscler Thromb Vasc Biol. 2000; 20(5): 1262-1275, doi: 10.1161/01.atv.20.5.1262, indexed in Pubmed: 10807742.

24. Roleder T, Kovacic JC, Ali Z, et al. Combined NIRS and IVUS imaging detects vulnerable plaque using a single catheter system: a head-to-head comparison with OCT. EuroIntervention. 2014; 10(3): 303-311, doi: 10.4244/EIJV10I3A53, indexed in Pubmed: 24769522.

25. Stone GW, Maehara A, Lansky AJ, et al. A prospective naturalhistory study of coronary atherosclerosis. N Engl J Med. 2011; 364(3): 226-235, doi: 10.1056/NEJMoa1002358, indexed in Pubmed: 21247313.

26. Cheng JM, Garcia-Garcia HM, de Boer SPM, et al. In vivo detection of high-risk coronary plaques by radiofrequency intravascular ultrasound and cardiovascular outcome: results of the ATHEROREMO-IVUS study. Eur Heart J. 2014; 35(10): 639-647, doi: 10.1093/eurheartj/eht484, indexed in Pubmed: 24255128.

27. Glagov S, Weisenberg E, Zarins CK, et al. Compensatory enlargement of human atherosclerotic coronary arteries. N Engl J Med. 1987; 316(22): 1371-1375, doi: 10.1056/NEJM198705283162204, indexed in Pubmed: 3574413.

28. Gardner CM, Tan H, Hull EL, et al. Detection of lipid core coronary plaques in autopsy specimens with a novel catheter-based near-infrared spectroscopy system. JACC Cardiovasc Imaging. 2008; 1(5): 638-648, doi: 10.1016/j.jcmg.2008.06.001, indexed in Pubmed: 19356494.

29. Schuurman AS, Vroegindewey M, Kardys I, et al. Near-infrared spectroscopy-derived lipid core burden index predicts adverse cardiovascular outcome in patients with coronary artery disease during long-term follow-up. Eur Heart J. 2018; 39(4): 295-302, doi: 10.1093/eurheartj/ehx247, indexed in Pubmed: 28531282.

30. Oemrawsingh RM, Cheng JM, García-García HM, et al. Nearinfrared spectroscopy predicts cardiovascular outcome in patients with coronary artery disease. J Am Coll Cardiol. 2014; 64(23): 2510-2518, doi: 10.1016/j.jacc.2014.07.998, indexed in Pubmed: 25500237.

31. Kini AS, Baber U, Kovacic JC, et al. Changes in plaque lipid content after short-term intensive versus standard statin therapy: the YELLOW trial (reduction in yellow plaque by aggressive lipid-lowering therapy). J Am Coll Cardiol. 2013; 62(1): 21-29, doi: 10.1016/j.jacc.2013.03.058, indexed in Pubmed: 23644090.

32. Sachdeva A, Cannon CP, Deedwania PC, et al. Lipid levels in patients hospitalized with coronary artery disease: an analysis of 136,905 hospitalizations in Get With The Guidelines. Am Heart J. 2009; 157(1): 111-117.e2, doi: 10.1016/j.ahj.2008.08.010, indexed in Pubmed: 19081406.

33. Nagasawa Y, Shinke T, Toh R, et al. The impact of serum trans fatty acids concentration on plaque vulnerability in patients with coronary artery disease: Assessment via optical coherence tomography. Atherosclerosis. 2017; 265: 312-317, doi: 10.1016/j. atherosclerosis.2017.06.922, indexed in Pubmed: 28697847. 
34. Rosenson RS, Brewer HB, Davidson WS, et al. Cholesterol efflux and atheroprotection: advancing the concept of reverse cholesterol transport. Circulation. 2012; 125(15): 1905-1919, doi: 10.1161/ CIRCULATIONAHA.111.066589, indexed in Pubmed: 22508840.

35. Navab M, Reddy ST, Van Lenten BJ, et al. HDL and cardiovascular disease: atherogenic and atheroprotective mechanisms. Nat Rev Cardiol. 2011; 8(4): 222-232, doi: 10.1038/nrcardio.2010.222, indexed in Pubmed: 21304474.

36. Honda S, Sidharta SL, Shishikura D, et al. High-density lipoprotein cholesterol associated with change in coronary plaque lipid burden assessed by near infrared spectroscopy. Atherosclerosis. 2017; 265: 110-116, doi: 10.1016/j.atherosclerosis.2017.08.016, indexed in Pubmed: 28881268.

37. Park SJ, Kang SJ, Ahn JM, et al. Effect of statin treatment on modifying plaque composition: a double-blind, randomized study. J Am Coll Cardiol. 2016; 67(15): 1772-1783, doi: 10.1016/j. jacc.2016.02.014, indexed in Pubmed: 27081016.
38. Hong MK, Park DW, Lee CW, et al. Effects of statin treatments on coronary plaques assessed by volumetric virtual histology intravascular ultrasound analysis. JACC Cardiovasc Interv. 2009; 2(7): 679-688, doi: 10.1016/j.jcin.2009.03.015, indexed in Pubmed: 19628193 .

39. Campeau L, Enjalbert M, Lespérance J, et al. The relation of risk factors to the development of atherosclerosis in saphenousvein bypass grafts and the progression of disease in the native circulation. A study 10 years after aortocoronary bypass surgery. N Engl J Med. 1984; 311(21): 1329-1332, doi: 10.1056/ NEJM198411223112101, indexed in Pubmed: 6333635.

40. Danek BA, Karatasakis A, Alame AJ, et al. Saphenous vein graft near-infrared spectroscopy imaging insights from the lipid core plaque association with clinical events near-infrared spectroscopy (ORACLE-NIRS) registry. Catheter Cardiovasc Interv. 2017; 89(6): E172-E180, doi: 10.1002/ccd.26696, indexed in Pubmed: 27535486. 